Borrelli, A.

\title{
The making of an intrinsic property: "Symmetry heuristics" in early particle physics
}

Journal article | Accepted manuscript (Postprint)

This version is available at https://doi.org/10.14279/depositonce-8372

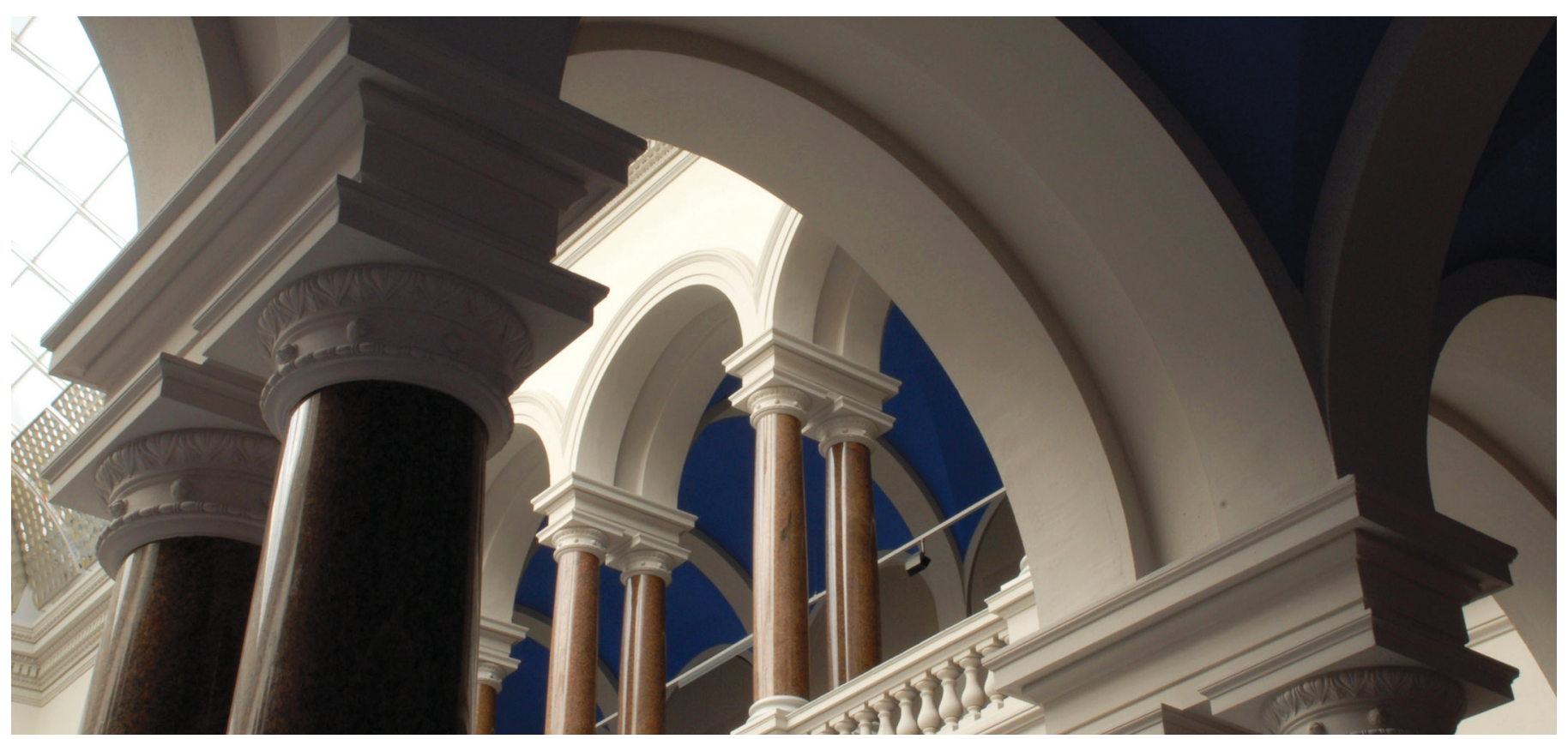

Borrelli, A. (2015). The making of an intrinsic property: 'Symmetry heuristics' in early particle physics.

Studies in History and Philosophy of Science Part A, 50, 59-70.

https://doi.org/10.1016/j.shpsa.2014.09.009 
Arianna Borrelli (Technische Universität Berlin)

\section{The making of an intrinsic property: "symmetry heuristics" in early particle physics}

(Published in Studies in the History and Philosophy of Science, 50 (2015) 59-70)

\section{Abstract}

Mathematical invariances, usually referred to as "symmetries", are today often regarded as providing a privileged heuristic guideline for understanding natural phenomena, especially those of micro-physics. The rise of symmetries in particle physics has often been portrayed by physicists and philosophers as the "application" of mathematical invariances to the ordering of particle phenomena, but no historical studies exist on whether and how mathematical invariances actually played a heuristic role in shaping microphysics. Moreover, speaking of an "application" of invariances conflates the formation of concepts of new intrinsic degrees of freedom of elementary particles with the formulation of models containing invariances with respect to those degrees of freedom. I shall present here a case study from early particle physics (ca. 1930-1954) focussed on the formation of one of the earliest concepts of a new degree of freedom, baryon number, and on the emergence of the invariance today associated to it. The results of the analysis show how concept formation and "application" of mathematical invariances were distinct components of a complex historical constellation in which, beside symmetries, two further elements were essential: the idea of physically conserved quantities and that of selection rules. I shall refer to the collection of different heuristic strategies involving selection rules, invariances and conserved quantities as the "SICtriangle" and show how different authors made use of them to interpret the wealth of new experimental data. It was only a posteriori that the successes of this hybrid "symmetry heuristics" came to be attributed exclusively to mathematical invariances and group theory, forgetting the role of selection rules and of the notion of physically conserved quantity in the emergence of new degrees of freedom and new invariances. The results of the present investigation clearly indicate that opinions on the role of symmetries in fundamental physics need to be critically reviewed in the spirit of integrated history and philosophy of science. 


\section{Introduction}

Mathematical invariances and group-theoretical structures, which are usually referred to collectively as "symmetries", have special prominence in today's theoretical physics. In the last decades they have also increasingly often attracted the attention of philosophers of science who see them as providing a privileged guideline for knowledge construction and possibly also for ontological reflection (Brading\&Castellani 2003, Debs\&Redhead 2007, French 1999, 2000, Lyre 2012, van Fraassen 1989). The present study focuses on the alleged function of symmetries in the construction of physical knowledge, a function which I follow previous authors in characterizing as "heuristic". I address the issue in the spirit of integrated history and philosophy of science by means of a case study from early particle physics.

While there is a general agreement that symmetries have become particularly significant in the context of twentieth century science, and especially particle physics (Michel 1989, 377. Schweber 2003, 386), so far no historical study has reconstructed how they came to play such a prominent role and whether that development was really linked to their special heuristic power, as often claimed a posteriori. Using the term "symmetry heuristics" to indicate the complex of heuristic strategies which led to the rise of mathematical invariances in particle physics, I will try to assess how those strategies actually looked like by analysing the emergence of the concept of "baryon number" as a conserved, intrinsic property of particles and of the invariance today associated to it. I will argue that in this process mathematical invariances were only one aspect of a heuristic constellation in which two further elements were essential: the ideas of physically conserved quantities and of selection rules. It was only a posteriori that the successes of this hybrid symmetry heuristics came to be attributed exclusively to mathematical invariances. After a brief discussion of the research questions and of the thesis of the paper (sections 2 and 3) and an introduction to the history of invariance and conservation (section 4) and to baryon number (section 5), I shall expound in detail the case study (sections 6 to 11), summarizing and tentatively generalizing the results of the analysis in some concluding remarks (section 12).

\section{The "heuristic power of symmetry" in accounts of physicists and philosophers}

When discussing the heuristic power of symmetry, philosophers often state that "symmetry principles" played a role not only in modern science, but also in pre-modern natural philosophy, thus linking mathematical invariances to qualitative and aesthetic notions of "symmetry" and suggesting that no clear-cut distinction between the two obtains (Brading and Castellani 2003, 3-11, 13; Debs\&Redhead 2007, 53-55 and 67-68; Lyre 2012, 368-370; van Fraassen 1989 233-289). Giora Hon and Bernard Goldstein (2008) have criticized such general claims and convincingly 
argued that a notion of symmetry linked to mathematical invariance only emerged at the end of the 18 th century and that it is anachronistic to interpret the earlier works as "implicit" applications of the modern symmetry concept (Hon\&Goldstein 2008, 27-48). Accordingly, my discussion shall consider the heuristic role of mathematical invariances, and not of general aesthetic considerations. No historian has so far explored the role of mathematical invariances in early particle physics, but many physicists have reminisced about it. Steven Weinberg, for example, wrote:

"When I first started doing research in the late 1950s, physics seemed to me to be in a dismal state. [...] Nature, like an enemy, seemed intent on concealing from us its master plan. At the same time, we did have a valuable key to nature's secrets. The laws of nature evidently obeyed certain principles of symmetry, whose consequences we could work out and compare with observation, even without a detailed theory of particles and forces. There were symmetries that dictated that certain distinct processes all go at the same rate, and that also dictated the existence of families of distinct particles that all have the same mass. Once we observed such equalities of rates and masses, we could infer the existence of a symmetry, and this we thought would give us a clearer idea of the further observations that should be made, and of the sort of underlying theories that might or might not be possible. It was like having a spy in the enemy's high command" (Weinberg 2011).

Weinberg, like many other physicists, regards the alleged effectiveness of symmetry consideration as following from the fact that the laws of nature "evidently" obey symmetry principles. However, his statements on how these principles actually guide research have an ambiguous character which is shared by most physicists' recollections of the same events: on the one side, experimental data on particles are presented as chaotic and puzzling, with nature "intent to conceal to us its master plan", while on the other hand scientists could somehow conceive "evident" symmetry principles and then find in observations the "equalities of rates and masses" predicted by them. In the end it remains unclear how the symmetries became evident in the first place, since those same recollections underscore how complex and long the path from chaos to order was (e.g. Michel 1989, Ne'eman 1987).

Which heuristic strategies were actually used to go from chaos to order and how did invariances fit in them? Steven French has extensively discussed the heuristic function of mathematical invariances and group theory in the development of micro-physics (French 1999, 2000). Working within the philosophical framework of structural realism, French focusses on historical actors who explicitly employed invariances and/or group theory to extend already existing mathematical models, and leaves aside the issue of the emergence of such models. Although admitting that "theories and models do not spring up, inductively, from the humus of observation and experiment, nor do they simply 'pop' into existence out of the head of scientists", French searches for the "heuristic power of symmetry" only in intra-theoretical developments (French 
1999, 103-105). While this heuristic function of invariances is indisputable, it only covers a limited amount of the process of knowledge construction in quantum physics, although French seems to suggest that it may have been a main motor for the development of the discipline (French 2000, $113)$.

Debs\&Redhead (2007, 53-55), too, only discuss the "heuristic power" of symmetries for extending models. Brading and Castellani (2003) have a more differentiated approach to "status and significance" of symmetry in the modern physical sciences and distinguish four possible functions (classificatory, normative, unifying and explanatory), all of which are regarded as evidence that symmetries, i.e. mathematical invariances, have "an important heuristic function" and a "strong methodological status" (Brading\&Castellani 2003, 11-13, quote p. 13). Claiming that "the history of the application of symmetry principles in quantum mechanics and then quantum field theory coincides with the history of the development of twentieth-century theoretical physics" they quote four "salient aspects" of this history: the introduction of "local gauge symmetries" (i.e. space-time dependent phase transformations) in general relativity; their application to the "internal" degrees of freedom of elementary particles; the increasing importance of discrete symmetries like parity and the emergence of the notion of spontaneous symmetry breaking (Brading\&Castellani 2003, 8). In these examples the heuristic power of symmetry is once again equated to the employment of grouptheoretical methods in extending mathematical models, without asking how the models emerged in the first place. One issue is here of particular relevance: In today's theories, mathematical transformations are applied not only to the four space-time coordinates, but also to the so-called "internal" degrees of freedom of elementary particles, such as "flavour" or "colour". These degrees of freedom have no correspondence in the macroscopic world, but are today regarded as intrinsic physical properties of particles (Haywood 2011, Itzykson and Zuber 1980). How did these new particle properties come to be? Brading and Castellani deal with their origin only in a footnote:

"The starting point for the idea of internal symmetries was the interpretation of the presence of particles with (approximately) the same value of mass as the components (states) of a single physical system, connected to each other by the transformation of an underlying symmetry group. This idea $[\ldots]$ was in fact due to Heisenberg $[\ldots]$ who in a 1932 paper introduced the SU(2) symmetry connecting the proton and the neutron (interpreted as two states of a single system)" (Brading\&Castellani 2003, 7, note 9).

Once again, an "equality of mass" is allegedly observed and from there a symmetry is inferred - but did the historical process really take this form? In 1932 Heisenberg indeed represented protons and neutrons by means of the "spin up" and "spin down" indices of the same field-symbol, yet he did not consider them as states of the same physical object, but rather interpreted neutrons as a composite of protons and electrons (Brown 1988). Moreover, Heisenberg did not postulate an 
invariance of nuclear interaction with respect to an exchange of neutron and protons and, although he made use of the Pauli matrices, he never mentioned SU(2). Finally and most importantly, isospin was not regarded as a physically relevant degree of freedom until its employment in nucleon-pion scattering in the early 1950s. In short, it took two decades and a host of new theoretical and experimental developments for the "isospin" index to come to be regarded as a physical property associated to an invariance. If one reads into Heisenberg's 1932 papers the later notions, one is collapsing in one single step two decades of efforts at conceptualizing in new forms the incoming experimental results on particle phenomena and ignoring the heuristic tools which may have contributed to this process. I shall argue that the foregoing descriptions of "symmetries heuristics" in the construction of scientific knowledge do little justice to the actual research practices of early high energy physics. The history of baryon number discussed below offers a simple but significant example of how how the idea of a new degree of freedom could emerge from experimental results and how this process was at times linked to, but always distinct from the employment of invariances in theory construction.

\section{The heuristic constellation of selection rules, invariance and physical conservation ("SIC- triangle")}

The general thesis to be illustrated by means of the case study is that the process of knowledge construction resulting in the formulation of theories of elementary particles in which mathematical invariance have a central role was due to a heuristic constellation in which those invariances were only one component. To understand the heuristic strategies behind these developments two further elements have to be taken into account: the notion of physically conserved quantity and that of selection rules. As shall be discussed more in detail in the next section, these two notions emerged respectively in the late $19^{\text {th }}$ century (physically conserved quantities) and early $20^{\text {th }}$ century (selection rules) independently both from each other and from mathematical invariances. In the late 1920s many-fold connections between selection rules, mathematical invariances and physically conserved quantities emerged in context of the study of atomic, molecular and nuclear spectroscopy, and around 1950 some authors started employing the three notions and their connections as heuristic tools for studying particle phenomena. This heuristic constellation became a key factor in a long, collective process of knowledge construction which led to the emergence of the concept of "internal" degrees of freedom and of the "internal symmetries" associated to them. It was not an application of invariances to interpret phenomena, but a process of co-construction of phenomena and theory, a process from which first new particle degrees of freedom and then new invariances emerged. The case of baryon number will offer a simple, but significant example of how the process 
developed. However, before going on with the case study, the heuristic constellation composed by selection rules, invariances and conserved quantities has to be introduced, and for simplicity this will be done by first looking at it as it appears in today's particle physics and then taking a look back in time at its development until the 1920's.

Let us first consider physically conserved quantities and their link to invariances. Internal quantum numbers such as isospin or colour are today regarded as representing physical properties of particles. Some of these properties are "physically conserved" in the sense that they are passed on by a particle to its decay products, while others are physically conserved only in processes due to some specific interaction. Each physical conservation is expected to have a mathematical expression in the relevant theory and, as we shall see later on, classical and quantum systems may have different mathematical forms corresponding to the same physical conservation. These conserved mathematical forms ("constants of motion") can in turn be connected to the mathematical invariances of the relevant theory. However, the idea of a physically conserved quantity must be regarded as distinct from that of a constant of motion both historically and philosophically. As we shall see in the case study, the template for conceiving physically conserved quantities in early particle physics was electric charge.

Now let us consider selection rules: The phenomena dealt with by particle theory are almost exclusively scattering and decay processes, which are conceived as transitions from an initial state (A) to a final state (B), where A and B may or may not contain the same particles. Quantum theory does not provide formal descriptions of transitions between states, but only gives estimates of their probability. "Selection rules" are expressions stating more or less generic principles according to which certain transitions may or not occur. These principles may be determined either bottom-up or top-down: If a certain transition is never observed, one may formalize this empirical result by stating that a selection rule forbids it, while on the other hand a theory may allow to derive selection rules which can be tested experimentally. In quantum mechanics and quantum field theory selection rules can follow from invariances. For example, an interaction which does not change under space reflection cannot connect states which behave differently under that transformation. Selection rules can also be regarded as resulting from a physically conserved quantity, because transitions between states having different values of it clearly cannot happen. It is important to note that the link between selection rules and physically conserved quantities is valid also if no relevant mathematical theory can be formulated.

In conclusion, there are today many ways of mutually connecting selection rules, mathematical invariances and physically conserved quantities and we may schematically represent all these possibilities as forming a triangular constellation "SIC-triangle" (fig. 1). Each of the 
corners of the SIC-triangle represents an independent heuristic strategy: conceptualizing experimental results in terms of selection rules, postulating the existence of a physically conserved quantity and employing mathematical invariances in the construction of new models. The special heuristic power of the SIC-triangle however resides in the fact that these three strategies may be variously connected to each other to enhance their potential: each arrow represents one further heuristic path combining two of the notions, as for example postulating a conserved quantity to explain a selection rule or testing an invariance by deriving a selection rule from it. The scheme is not meant as a summary of all possible heuristic strategies used in (early) particle physics, but it is my claim that it subsumes all those strategies in which mathematical invariances were used to interpret experimental results or formulate testable predictions. I shall argue that from the late 1940s onward physicists increasingly often conceptualized (new) experimental results in terms of selection rules and, to a lesser extent, physically conserved quantities. From there, the various heuristic paths of the SIC-triangle could be fruitfully employed, although at first not all authors exploited the full possibilities of the triangle. Only on this basis could invariances come to play a role in model-building. By the end of the 1950s, selection rules where recognized as the phenomenological entry-point to learn about the mathematical invariances and physical conservations of particle interactions, as explained in one of the earliest overviews on "Elementary particles and symmetry principles":

"The initial problems for [particle] theory fall into two main categories: We want to understand (a) selection rules and (b) lifetimes or branching ratios, and cross sections. [...] The [following] discussion is directed principally to the primary problem of selection rules [...]The goal is to attain a 'complete selection-rule scheme', i.e., a theoretical scheme with the following two properties:

(1) No interaction which is observed is completely forbidden by the scheme.

(2) All interactions which remain unobserved after being carefully sought after are accounted for as forbidden (at least in low order).

[...] We can expect that a complete selection-rule scheme will result from knowing all the symmetry principles which hold for system of interacting elementary particles [...] A very important alternative way of looking at selection rules, one which is much beloved of physicists because it lends itself easily to intuitive formulations, is in terms of conservation principles. When we say that a system obeys a particular conservation principle we instinctively think of an entity associated with the system, which has the 'substance-like' property of keeping its magnitude constant in time. The bearing on selection rule is that the system may evolve only into states associated with the same value of the conserved entity as it had initially "(Melvin 1960, 480-481, italics in the original).

This passage explains how the notion of selection rules allowed to conceive both the observation and the non-observation of certain phenomena as evidence about the invariances and/or conserved quantities of a theory. This research practice was therefore no simple "observation" of "equalities of 
rates and masses", but was rather a complex process of combined construction and conceptualization of phenomena requiring the employment of the whole SIC-triangle.

\section{The emergence of the connection between invariance, conservation and selection rules}

Today it seems straightforward to conceive constants of motion of classical mechanics as representing conserved physical quantities such as energy or momentum, but a look back at historical developments shows how this identification is all but trivial. As I have argued in detail in the case of angular momentum (Borrelli 2011), relationships between the invariances of the equations of a system and constants of motion were known at least since 1800, but they were not regarded as having a greater physical significance than other formal properties of analytical mechanics. It was only after the principle of energy conservation became established in the late 19th century that mathematically defined "constants of motion" slowly came to be regarded as representing physical properties which are conserved also beyond the limits of validity of specific mathematical models, as is the case with energy. In context of a discussion on whether and how the physical principle of energy conservation could be mathematically expressed in general relativity Emmy Noether (1918) developed two general theorems linking invariances to constants of motions in classical (relativistic) Lagrangians. These theorems are today often regarded by philosophers of science as summing up the issue of symmetries and conservation (Brading and Brown 2003). As the previous discussion should have made clear, though, this view is reductive and uncritically equates physical notions to mathematical structures and vice versa. Noether's theorems are purely mathematical results and say nothing about the existence and properties of physically conserved quantities associated to the constants of motion. Moreover, they do not even provide a complete mathematical basis to discuss invariances in all physical theories. ${ }^{1}$ The text by Melvin quoted above gave a good description of how physicists like to think of conservation: "When we say that a system obeys a particular conservation principle we instinctively think of an entity associated with the system, which has the 'substance-like' property of keeping its magnitude constant in time." (Melvin 1960, 481). It is not relevant to our discussion whether such views can be associated with a philosophically viable view of reality or not: it is a fact that they play a key role in scientific practice, as the case study will show. In conclusion, constants of motion and physically conserved quantities are epistemologically distinct elements of a heuristic constellation.

While Emmy Noether developed her theorems, quantum theory was already arising and it was in that context that the notion of selection rules arose (Borrelli 2009). In the old quantum

1 Noether's theorems are not applicable to non-relativistic quantum mechanics and, due to the issue of renormalization, they are not sufficient to formalize the question in quantum field theory (Itzykson\&Zuber 1980, 509-561). 
theory (1913-1925) the discrete spectra of atoms were interpreted in terms of transitions between discrete energy levels, and the empirical rules stating which of the possible transitions did or did not occur were known as "selection rules". After the emergence of quantum mechanics in 1926-27 Eugene Wigner showed how the invariances of Schrödinger's equation could be used to explain selection rules. However, at that point it remained unclear whether and how physically conserved quantities as known from classical theory could be conceived in quantum terms. This problem was solved by Wigner in an essay on "Conservation principles in quantum mechanics" ("Erhaltungssätze in der Quantenmechanik", 1928). Wigner started by noting that in quantum mechanics it made no sense to ask what value a certain observable would have, but only the probability with which this or that value might be measured. This applied also to classically conserved quantities such as energy, so that no classical conservation law could obtain. One needed, so Wigner, "to formulate in this [probabilistic] sense also the conservation laws. They will then state that for example the probability that the energy has the value E does not change in time". ${ }^{2}$

Despite its simplicity, this statement was of paramount physical significance: it acknowledged that, even in the case of energy, the classical notion of a "conserved quantity" as a quantifiable, constant property of an isolated system was not applicable and it introduced a quantum version of conservation. Wigner then showed that his notion of physical quantum conservation formally corresponded to requiring that the mathematical operator representing the quantum-conserved quantity would leave invariant the Schroedinger equation of the system. Thus, in quantum mechanics, too, a mathematical connection between invariances and physically conserved quantities could be established, although no constants of motion existed. This correspondence was extremely important to strengthen the impression that, despite the dramatic formal and empirical differences between classical and quantum mechanics, the two theories could still be regarded as referring to the "same" physical quantities, such as energy or momentum. It is significant that Wigner's highly innovative proposal for quantum conservation was rapidly and quietly taken over by the scientific community, although his paper was rarely if ever quoted: a sign that the construction helped fill a perceived gap between the classical and quantum approaches, allowing physicists to "intuitively" grasp selection rules in terms of "substance-like" conserved entities, as noted by Melvin (1960). Wigner then went on to use his previous results on the link between the invariances of Schroedinger's equations and selection rules to show that the latter provided in quantum theory the observable signatures of conservation laws. His formalism also allowed to define a conserved quantity - today known as parity - which played no role in classical physics, but manifested itself in

2 "In diesem Sinne müssen wir auch die Erhaltungsätze formulieren. Sie lauten dann z. B. Die Wahrscheinlichkeit, daß die Energie den Wert E hat, ändert sich im Laufe der Zeit nicht” (Wigner 1927, 375). 
the quantum world through a selection rule which had been assessed years earlier by Otto Laporte (Wigner 1928, 378).

Wigner was the first to exploit the constellation which I termed "SIC-triangle", and this heuristic method was quickly taken up by other physicists and put to good use in analysing the spectra of molecules and, later on, of atomic nuclei. In the late 1940s the SIC-triangle was employed in particle physics by some authors who noted that space-time invariances and the related conservations laws (especially parity and angular momentum) led to selection rules for particle decay and interactions (Michel 1989, 373-374). Yet this was not all: the absence of certain particle phenomena was eventually conceptualized in terms of selection rules due to new conserved quantities, and this was the first step towards the emergence of internal quantum numbers and symmetries. In the following pages the emergence of baryon number will be discussed as an example.

\section{Baryon number and its "origins"}

Baryon number is perhaps the simplest among the internal quantum numbers of particle theory (Haywood 2011, 47, 49). Only strongly interacting fermions ("baryons") have non-zero baryon number, conventionally set to +1 for baryons and -1 for antibaryons. All other particles have baryon number equal to zero. Baryon number is today regarded as strictly conserved and its best known observable consequence is a selection rule forbidding the decay of the proton, the lightest baryon. Baryon number has no connection with the charge of strong interactions, which is "colour". Finally, baryon number conservation is associated to the invariance of the Standard Model with respect to a phase transformation of any field $\psi$ having the form:

$\psi \rightarrow \exp (\mathrm{iB} \alpha) \psi$

where $\mathrm{B}$ is the baryon number of $\psi$ and $\alpha$ is an arbitrary constant. Both the formalism and the physical significance of baryon number conservation appear today almost trivial and deserve only a passing mention in textbooks. Scientists tend to conceive this notion as a long-standing component of particle theory. In his overview of the development of micro-physics Abraham Pais connected the "faint first glimpse of this conservation principle" to a paper by Hermann Weyl (1929), adding that "it was Stueckelberg who, in 1938, formulated the principle in a more realistic setting"' (Pais 1986, 488). Louis Michel, in his recollections on "Symmetry and conservation laws in particle physics in the fifties" (1989) writes: "If I had been asked a few months ago about the conservation law for nucleons and its consequences for antiproton production, I would have answered that this was well understood from Dirac's 1931 paper” (Michel 1989, 380). However, reading the original papers he 
had had to acknowledge that the law emerged much later, perhaps as late as 1952. In the following sections I shall show how the components of the SIC-triangle were variously exploited as heuristic tools, allowing to interpret a broad range of observed and non-observed phenomena in terms of a new conservation law associated with a new mathematical invariance.

\section{The SIC-triangle before World war II: Weyl (1929) and Stückelberg (1938)}

In 1928 Paul Dirac formulated his relativistic equation for a four-component electron field, noting that it had solutions of potentially unlimited negative energy which were difficult to interpret physically (Pais 1986, 347-348). In 1929 Weyl suggested that two of the four components of Dirac's field could be interpreted as electrons, the other two as protons. However, with this interpretation the Dirac equation would allow processes in which a proton and an electron mutually annihilated, a prediction contradicting the observed stability of matter. To make his theory compatible with experiment, Weyl added to it an ad-hoc selection rule forbidding electron-proton annihilation by assuming the separate conservation of positive and negative electric charge. He formally implemented this physical conservation by means of two distinct local gauge invariances, one for positive, one for negative charge (Weyl 1929, 332). What seems today as a radical (and empirically untenable) hypothesis, i.e. the separate conservation of positive and negative electric charges, was at the time still experimentally viable, so that Weyl had no need to conceive a thoroughly new conserved quantity when employing the SIC-triangle. All three elements of the SIC-triangle were used: the absence of proton-electron annihilation was conceptualized as evidence for a selection rule which in turn led to theory modification by means of a new physical conservation law (of positive and negative charges) and of a new invariance associated to it. However, Weyl was not trying to find new symmetries of nature, but only attempting to save both the phenomena and his theory. Whether or not his work should be regarded as "the first glimpse" of baryon number conservation, as Pais claims, remains a matter of taste.

In the 1930s the first "new", "unstable" particles were observed: the neutron (1932), the positron (1931-33) and a particle with mass intermediate between electron and proton, the "mesotron" or "meson" (1937), which soon came to be interpreted as the particle mediating nuclear interactions proposed in 1935 by Hideki Yukawa (Pais 1986, 351-353, 397, 429-436). Scientists faced evidence that many more particles existed than originally assumed and that they could variously transform into each other, raising the question of the laws according to which the transformations happened. No distinction was made at first between the forces responsible for the cohesion atomic nuclei (today known as "strong" interactions) and the ones involved in beta decay (today known as "weak" interactions), but it was becoming clear that these phenomena were not of 
electromagnetic nature.

Among the first theorists to react to these developments was Ernst Stückelberg, working in Geneva. Stückelberg developed a quantized field theory encompassing both electrodynamics and the recently discovered interactions of "heavy" particles (neutrons and protons), both of which he regarded as mediated by vector bosons: the photon and Yukawa's meson (Stückelberg 1938a, 1938b). He used electromagnetism as a template for developing a field theory of heavy-particle interactions, determining the form of the couplings by assuming the existence and conservation of a "heavy charge" analogous to the electric one. As evidence for this new physically conserved quantity he quoted the non-observation of heavy particles decaying into light ones:

"Apart from the [...] conservation law of electric charge there must clearly be another one: in all observed transformations of matter, none have been observed where heavy particles (neutrons and protons) transformed into light ones (electrons and neutrinos). We will therefore require a law of conservation of heavy charge" (Stückelberg 1938b, 317 , italics in the original). ${ }^{3}$

To formally derive the couplings from heavy-charge conservation Stückelberg assumed the validity of a "continuity equation" ("Kontinuitätsgleichung") for heavy charge density analogous to the one for electric charge (Stückelberg 1938b, 218-219). Although this equation can in principle be regarded as following from an invariance of the Lagrangian, Stückelberg did not present it as such, but instead saw it as expressing the physical requirement that the flow of heavy charge should be conserved like electric current was. In this case, only two corners of the SIC-triangle were present: a selection rule conceptualizing the non-observation of proton decay and a new physically conserved quantity. The existence of the conserved quantity was not deduced on the basis of the selection rule, but independently postulated in analogy to electric charge. However, the connection between the assumed selection rule and the postulated conserved heavy charge served to mutually reinforce the two hypotheses.

\section{Early developments after WW2 (1949): Okayama' "mesic charge" and Wigner's reflections on invariance and the "law of conservation of fheavyheavy particles"}

As we saw in the previous section, already before the Second World War the stability of matter had been conceptualized in terms of a selection rule associated to a conserved quantity identical with or analogous to electric charge. During the war research in physics slowed down, apart from those areas relevant to the war effort and the further papers relevant to our subject were written after the

3 “Ausser diesem [...] Erhaltungssatz der elektrischen Ladung, gibt es aber offenbar noch einen weiteren Erhaltungssatz: Bei allen beobachteten Umwandlungen der Materie, wurden noch keine Umwandlungen von schweren Partikeln (Neutron und Proton) in leichte Partikeln (Elektron und Neutrino) beobachtet. Wir wollen daher einen Erhaltunggsatz der schweren Ladung fordern" (Stückelberg 1938b, 317). 
end of the war, in a much changed academic and geopolitical climate. During the Cold War years the USA started a long-running investment campaign in experimental and theoretical research in particle physics. Impressive results were soon achieved on the experimental front, with the realization in 1947 that what we today call muons and pions were two different particles and, soon after that, the indications from cosmic rays and accelerator experiments that even more particles and interactions existed. While experimental activity was concentrated in Britain and the US, already by 1948 contributions to theory also came from Japanese scientists, who showed great resilience in the aftermath of the Second World War (Konuma 1989). Among these contributions was a short letter to Physical Review by Taisuke Okayama with the title "On the mesic charge" (1948).

Like Stückelberg, Okayama, too, attempted to formulate a field theory of strong (in his terminology "mesic") interactions modelled on electromagnetism, with couplings proportional to a "mesic" charge and interactions mediated by a "mesic" field analogous to the photon. ${ }^{4} \mathrm{He}$ made two assumptions: (1) mesic charge was physically conserved and (2) nucleons satisfied the same Dirac equation valid for electrons, with holes representing nucleons having a "mesic charge" opposite to that of protons and neutron. Okayama employed the assumed physical conservation of mesic charge to restrict the possible form of interactions between nucleons and the mesic field. Mesic charge conservation was formalized as an invariance of the interaction such that "a hole in the negative energy state of the nucleon can be considered as a nucleon with the inverse mesic charge in the positive energy state [...] If there are really mesic charges with a charge conservation theorem, then many types of interaction between nucleons and mesic field can be excluded. Further, one may expect the existence of cascade showers of the hard component of the cosmic rays in the very high energy region. Further research on these will be reported elsewhere" (Okayama 1949, 308). Okayama exploited two components of the SIC-triangle: a physically conserved quantity and invariances. He also hinted at observable consequences of the model, but he seems never to have published again on the subject.

Okayama's work was rarely quoted, but among the few who referred to it was Eugene Wigner (1949), who mentioned it in his paper on "Invariance in physical theory" presented at the celebrations for Einstein's 70th birthday in Princeton. Wigner's text is perhaps the earliest one to offer a serious discussion of the role of invariances in research practice and it deserves much attention. Wigner underscored the importance of "invariances" in the search for the laws of nature, but made clear that for him mathematical invariances were significant only insofar as they expressed some physical principle of invariance: thus, the "older" invariances of classical

4 The mesic field was presumably conceived as associated to Yukawa's meson, but Okayama did not say so in his short letter, which containes no bibliographic references whatsoever. 
mechanics and special relativity were for him "the products of experience rather then a priori truths", because they expressed the observed physical irrelevance of position and orientation in space-time for the laws of mechanics and electromagnetism (Wigner 1949, 521-522). The "new" principles of invariance introduced by general relativity were for Wigner a substitute for the old ones and had a bolder, but equally physical significance: "information relating to distant points cannot add anything relevant to the knowledge of local conditions" (Wigner 1949, 522-523). With these strong phenomenological views on principles of invariance Wigner went on to speak of "invariance in quantum mechanics", noting how the "older principles of invariance are in harmony with quantum mechanics" and had a heuristic function also thanks to the physical conservation laws linked to them (Wigner 1949, 523). ${ }^{5}$ Wigner concluded the paper by discussing the conservation of electric charge which for him was "a dissonant sound":

"While the conservation laws for all other quantities, such as energy or angular momentum, follow in a natural way from the principles of invariance, the conservation law for electric charge so far has defied all attempts to place it on an equally general basis" (Wigner 1949, 524).

Wigner quoted the physical interpretation of local gauge invariance as the irrelevance of the absolute magnitude of electric potential, but deemed it physically unsatisfactory, because it could not be considered as a "product of experience" (Wigner 1949, 524-525). In other words, Wigner did not regard a mathematical invariance as physically significant just because it led to empirically successful predictions, and used the term "principle of invariance" only to indicate physical principles. He gave no definition of such principles, but various examples, such as the invariance of the laws of nature by a physical rotation or translation of a system. He therefore distinguished between electric charge as a physically conserved quantity and as a "constant of motion". This point is of great importance for the present subject, because by underscoring this gap Wigner came to wonder whether "similar conservation laws for other types of interactions" might exist (Wigner 1949, 525). He developed these reflections in a footnote at the end of the paper, where he stated:

"It is conceivable, for instance, that a conservation law for the number of heavy particles (protons and neutrons) is responsible for the stability of the protons in the same way as the conservation law for charges is responsible for the stability of the electron. Without the conservation law in question, the proton could disintegrate, under emission of a light quantum, into a positron, just as electrons could disintegrate, were it not for the conservation law for the electric charge, into a light quantum and a neutrino. (Wigner 1949, 525-526 note 9).

When reading this passage it is important to note that, although a posteriori it might appear

5 Wigner did not quote his own 1928 paper on the subject and, when giving references for classical mechanics, he did not mention Emmy Noether (Wigner 1949, 523, note 4). 
straightforward to ask why the proton is stable once the existence of the positron is known, there is no evidence that the question had been asked before. Indeed, historical sources suggest that the production and annihilation of protons and antiprotons had until then always been conceived in terms of pair production and annihilation (McConnel 1946). Stückelberg had regarded the stability of matter as an unproblematic fact supporting his theory of heavy-particle interactions, Wigner instead considered the selection rule forbidding proton decay as an explanandum whose physical cause had to be investigated. Although he suggested an analogy between electric charge and the charge of heavy particles interactions ("mesonic charge"), this connection was only one of the possibilities he mentioned. Among other possible explanations was for example the existence of a fundamental length. In this paper the whole SIC-triangle appeared: physically conserved quantities, mathematical invariances and selection rules. These elements and their connections were employed to postulate the existence of a thoroughly new conserved quantity which might or might not be the "charge" of heavy-particle interactions. Wigner's paper is of particular importance for understanding "symmetry heuristics" in early particle physics, since comparing it to the work of previous authors makes evident how the assumptions of the existence of a thoroughly new particle property was a highly innovative step resulting from reflection, and not a straightforward conclusion to be drawn immediately on the basis of empirical data.

\section{Embedding the "conservation of heavy particles" in mathematically refined models (1950):}

\section{Louis Michel's classes of particle phenomena and Yang and Tiomno's extension of parity}

The French theorist Louis Michel started his career in the late 1940s working with Leon Rosenfeld at the University of Manchester, and in 1950 published a paper where he attempted to construct a Hamiltonian subsuming a broad range of recent observations on beta and muon decay (Michel 1950a). Starting from the transformation properties of spinors with respect to their known degrees of freedom (space-time, electric charge, permutations and the charge of the non-electromagnetic interaction), Michel considered all invariant Hamiltonians that could be built and, using group theory, derived from them all possible selection rules. He also referred to Okayama's paper (Michel 1950a, 520). His method allowed to subdivide particle processes into "classes" in such a way that, if one process in a class was forbidden by a given selection rule, all other processes in the class would be forbidden, too. He was therefore employing two elements of the SIC-triangle: selection rules and invariances, as well as their connection.

A short time later, in a note sent to Nature, Michel used the idea of "classes" of processes to broaden the scope of the conservation of heavy particles (Michel 1950b). He suggested that a number of (observed and non-observed) processes involving nucleons could be grouped together in 
a "class" comprising (a) reactions making all atomic nuclei unstable (e.g. annihilation of protons and neutrons into pions), (b) the emission of antiprotons by a nucleus and (c) processes which implied the non-conservation of the number of nucleons. Since most nuclei are stable, he concluded that all the other processes had to be forbidden, too, noting that this idea could be used to study "mesic charge". ${ }^{6}$ In this second paper Michel did not derive selection rules from invariances, but instead worked bottom-up, formalizing the observation and non-observation of a broad range of phenomena in terms of a single new selection rules. Thus, Michel's two papers represented different research strategies contained in the SIC-triangle: the formal deduction of selection rules from invariances on the one side, and the construction on the basis of experimental data of more encompassing selection rules than those usually stated, on the other.

In 1950 Chen Ning Yang and Jayme Tiomno published a paper whose starting point was the remark that Dirac spinor fields, such as those representing nucleons, had four independent ways of changing under a space inversion, and not only two. The four possibilities corresponded to four "intrinsic parity" factors $(1,-1, \mathrm{i},-\mathrm{i})$ and, by assigning them to different particles and assuming their conservation, Yang and Tiomno derived both a proposal for a universal Fermi interaction and the "law of conservation of heavy particles which has been noticed by many physicists" (Yang\&Tiomno 1950, 497). To make their theory consistent with observed selection rules "some additional and rather arbitrary rules were introduced" to exclude specific terms from the interaction Hamiltonian (Yang\&Tiomno 1950, 497). Thus, Yang and Tiomno made use of all three elements of the SIC-triangle: invariances (under space inversion), physically conserved quantities (intrinsic parity) and selection rules (conservation of the number of heavy particles), also exploiting various connections between them.

Soon, however, Gian Carlo Wick, Eugene Wigner and Arthur Wightman (1952) pointed out that the assignment of particles to four intrinsic parity types (instead of two) could have no observable consequences and so could hardly be physically relevant. Nonetheless, they noted that one could formally associate the conservation of heavy particles to the invariance of interactions with respect to a phase transformation depending on an undetermined numerical factor which "had no immediate physical significance". The three authors stated: "It may be again desirable to keep this indeterminate phase as a useful formal device to remind one of this [i.e. heavy particle] conservation law." (Wick, Wightman\&Wigner 1952, 105). This statement is the earliest occurrence I could trace of a connection between the observed conservation of heavy particles and phase invariance (i.e. global gauge symmetry). The "indeterminate phase" represented the conservation of

6 In his recollection Michel claimed that at the time he knew neither about Okayama's work nor about Wigner's 1949 paper to be discussed in the next section, but this statement is only correct as far as Wigner's work is concerned (Michel 1989, 380). 
heavy particles as is formally done today, but was then regarded as having "no immediate physical significance", as it was not linked to any phenomenon other than the selection rule conserving heavy particles and was not connected to a conserved heavy-particle charge. Once again, Wigner (this time with co-authors) was not ready to grant physical significance to any mathematical invariance of a theory.

\section{Contributions in 1952: from the conservation of the number of heavy particles to a new intrinsic coordinate of particles.}

During the year 1952 a number of papers were published proposing new interpretations and/or extensions of the observed conservation of the number of heavy particles. Their authors came from different continents and scientific cultures: Pascual Jordan from West Germany, Leonard Schiff from the USA, where also the European emigrèes Pais and Wigner were based, Yakov Zeldovich from the USSR and Sadao Oneda from Japan. Each author had his own special perspective on the issue and not all of them knew of the parallel developments, but all of them exploited one or more of the heuristic strategies of the SIC-triangle. Later on, through the combination of the various perspectives, the notion of baryon number as a new intrinsic property distinct from strong charge eventually emerged.

\subsection{Jordan (1952): conservation of nucleonic charge and local gauge invariance}

During the war Pascual Jordan had remained in Germany and been a member of the Nazi party, so after the war he was at first outside of the academic system. In 1951, though, he took part in the celebrations for Werner Heisenberg's 50th birthday and presented a paper which took Wigner's Einstein tribute from 1949 as a starting point. Jordan's contribution had the title "On conservation principles in physics" (Über Erhaltungssätze in der Physik, 1952a) and began by stating that "the fundamental physical experience of the stability of matter must be regarded as strange and in need of explanation, since we know about the possibility of particle transformation, pair production and pair annihilation". 7 Thus, as in Wigner's paper, the non-observation of proton-electron annihilation became an explanandum.

Jordan noted that matter stability might be due to contingent causes, but claimed that it would be more satisfactory if it could be connected to a "specific, overarching conservation principle" and tentatively assumed that beside electric charge another kind of physically conserved

7 "Die physikalische Grunderfahrungen der Stabilität der Materie muß als etwas Merkwürdiges und der Deutung Bedürftiges angesehen werden, seitdem wir die Möglichkeit der Verwandlung und der Paarerzeugung oder Paarvernichtung von Elementarteilchen kennengelernt haben“ (Jordan 1952a, 78). 
charge existed. ${ }^{8}$ Noting that the connection between conservation principles and invariances was well-known, he proposed a model for nuclear interactions in which the "neutron charge" (which he claimed Wigner had introduced in his 1949 paper) was the conserved quantity associated to a local gauge invariance (Jordan 1952a, 78). Jordan implemented this general idea in a 6-dimensional theory, but one year later he derived what he regarded as the simplest model leading to the stability of matter (Jordan 1952b). The model involved a local gauge invariance associated to vector boson fields for nuclear interactions, in perfect analogy to electromagnetism. It is interesting to note how Jordan did not consider the simpler alternative of a global gauge invariance (i.e. a phase invariance), and it is plausible that he did so because he was closely following the physical example of electromagnetism, rather than the abstract structures of invariance. Nonetheless, Jordan was exploiting the whole SIC-triangle as a heuristic tool in his work: a selection rule formalized the stability of matter and linked it with a physically conserved quantity (neutron charge) and an invariance (local gauge invariance).

\subsection{Schiff (1952): Extending the "conservation of heavy particles"}

After 1950 the increasing flow of experimental data radically transformed the landscape of particle physics. Beside the two mesons already known, there was now clear evidence of other unstable particles: the so-called V-particles (Pais 1986, 512-523). The most puzzling feature of V-particles was the mismatch between their fast, copious production and their slow decay. Moreover, some of these particles were electrically neutral and heavier than protons, and were observed decaying into protons and negative mesons, but never into an anti-proton and a positive meson. ${ }^{9}$ The first one to reflect on the causes of this non-observation was the theorist Leonard Schiff (1952), at the time head of the Physics Department at Stanford University. Schiff explained the behaviour of Vparticles as follows:

"[T]here is a conservation law for all particles of nucleonic mass: protons, neutrons and V-particles, and other possible particles that decay into protons and neutrons, such as perhaps the charged V-particle. This is a simple extension of the generally accepted conservation law for protons and neutrons only, and may be stated as follows: the difference between the total number of nucleonic particles (protons, neutrons, Vparticles) and the total number of antinucleonic particles (negative protons, antineutrons, ant V-particles) is a constant of the motion" (Schiff 1952, 374).

Thus, Schiff extended the "conservation of heavy particles" to V's, and assumed that a heavy particle and its antiparticle had different properties in this respect. Yet he did not explicitly link

8 "besonderer, umfassend geltender Erhaltungssatz" (Jordan 1952a, 78).

9 Antiprotons had not yet been observed at the time, but there was in the physics community consesus about their existence and properties (Pais 1986, 488). 
these properties to a physically conserved quantity such as mesic charge, or to a mathematical invariance. Only one element of the SIC-triangle was present here: Schiff was conceptualizing the non-observation of a number of processes in terms of a single selection rule generalizing the one expressing matter stability. His reflections provided the starting point for other authors to exploit the SIC-triangle connect these non-observations to conserved quantities and/or invariances.

\subsection{Pais (1952): the "chemical" approach to particle theory}

The papers by Michel (1950b) and Schiff (1952) were examples of how theorists tried to develop bottom-up schemes to subsume different non-observations under the same selection rule without making any specific hypotheses on the physical causes behind it. A further development of this bottom-up approach was made necessary by the increasing complexity of observed particle phenomena, leading to the invention of elaborated "bookkeeping" schemes for particle processes. We have seen how the idea of the "stability of matter" had developed first into the "law of conservation of heavy particles" and then into the broader formulation given in 1952 by Schiff. In the same year Abraham Pais, at the time working at the Centre for Advanced Studies in Princeton, went one step further: Building upon suggestions made at a conference held in Tokyo in 1951, Pais constructed a simple mathematical model of the behaviour of V-particles which he presented at the second Rochester conference and in a subsequent influential paper (Messiah and Noyes 1952, 8995, Pais 1952). His main idea was to represent "heavy particles" (both nucleons and heavy V's) as $N_{i}$, with the index $i$ taking the value 0 for nucleons, and 1 for $\mathrm{V}$-particles. Mesons were indicated as $\pi_{i}$, with $i$ equal to 0 for pions and 1 for $\mathrm{K}$ mesons. All couplings had the form $N_{i} N_{j} \pi_{k}$ and Pais postulates that they would be strong if $i+j+k$ was even (fast production) and weak if it was odd (slow decay), thus providing a unified formalization of V-particles' properties. The form of the interaction implied necessarily that the number of $N$-type fields would be conserved in Schiff's sense, and Oppenheimer remarked that "this theory is [...] an extension of the old rule that the number of nucleons is conserved" (Messiah and Noyes 1952, 90). Pais later added that the old rule was also "itself an ad hoc assumption needed to guarantee sufficient stability of matter" (Pais 1952, 664). Pais did not to interpret his indices in terms of new properties of elementary fields and at the end of his paper he wrote:

"The search for ordering principles at this moment may indeed ultimately have to be likened to a chemist's attempt to build up the periodic system if he were given only a dozen odd elements" (Pais 1952, 672).

The reference to chemistry captures well the bottom-up practices some particle theorists were using at the time, in an "empiricist temper" which had come to dominate theoretical physics in the U.S.A 
(Schweber 1986). The main tool to conceptualize particle phenomena was to represent them as "reaction processes" in which a set of symbols was transformed into another one. The symbols could represent well-established particles (protons, electrons) but also new hypothetical entities defined by the reactions they took part in. By manipulating the symbols and arrows and tentatively matching them to theoretical hypotheses the absence of certain reactions could become positive evidence, with new particles and interactions emerging and old ones being transformed. The flexible, many-layered notion of "selection rules" played here a key heuristic role, connecting basic empirical statements ("the proton does not decay") to more general hypotheses ("heavy particles are conserved") and also abstract theoretical constructs (e.g. modified parity, Pais' indices). It must be noted that, while the notion of selection rule was at the core of Pais' idea, conserved quantities played no role and mathematical invariances were not developed, although Pais was very wellversed in the employment of group theory in theoretical research. The new indices introduced had a very ambiguous epistemic status which Pais chose not to discuss.

\subsection{Zeldovich (1952): "nuclear charge" as a new "intrinsic coordinate"}

In the following years, scientists from the Soviet influence area also took an interest in the conservation of heavy particles. The prominent physicist Yakov Zeldovich, best known for his work in nuclear theory and cosmology, wrote "On the theory of elementary particles. Conservation of the nuclear charge and a possible new type of V-particles" (1952). He built upon Schiff's results, conceptualizing them in terms of "nuclear charge":

"By analogy with the electric charge it seems reasonable to introduce the concept of nuclear charge and to ascribe to the proton and neutron an identical nuclear charge $+y$. The nuclear charge of $\pi$ and $\mu$ mesons, electrons, neutrinos and photons is equal to zero [...] the law of conservation of nuclear charge is an exact law like the law of conservation of electric charge" (Zeldovich 1952, 57, quoted from the English translation).

While acknowledging that he was only formalizing Schiff's ideas, Zeldovich noted that his approach allowed to unfold their full physical significance by making clear that a new intrinsic degree of freedom of particles was being postulated:

"The basic purpose of the present note is in fact to point out that, in addition to mass, spin, electric charge and parity there is yet another intrinsic coordinate of a particle - its nuclear charge. Due to the presence of such a coordinate we have the possibility of existence of two particles with the same mass, zero spin, and identical charge and magnetic moment, which nevertheless are strongly different, differing in the sign of the nuclear charge, i.e. differing in their decay products." (Zeldovich 1952, 58)

Thus, Zeldovich made explicit the transition from a conservation of the number of particles to the 
existence and conservation of a new "intrinsic coordinate". Although the term "nuclear charge" suggested the analogy to electric charge, Zeldovich did not say whether it should necessarily be linked to a particle's capability of nuclear interactions: His assignments were primarily derived "chemically" on the basis of allowed and forbidden reactions. In fact, Zeldovich soon extended his reflections to postulate the existence of a "neutrino charge", which was not linked to any new interaction, but only derived from the conservation of the difference between neutrinos and antineutrinos, a step taken independently also by the Hungarian theorist George Marx (Zeldovich 1953, Marx 1954a, 1954b). Like Wigner, Zeldovich not only exploited various heuristic possibilities offered by the SIC-triangle, but also explicitly discussed their physical implications, putting them at the core of his paper. His approach thus stood in clear contrast to that of Pais and other US-based theorists, who avoided explicit reflection on the physical implications of formal results.

\subsection{Wigner (1952): "neutronic charge" vs. "mesonic charge"}

In 1952 Wigner published further reflections "On the law of conservation of heavy particles", stating: "The purpose of this note is to trace more in detail the consequences of treating the conservation law of heavy particles on a par with the conservation law for electric charges" (Wigner 1952, 449). I this paper, Wigner was the first one to explicitly address the issue of the identification of a postulated new conserved quantity with the charge of heavy-particle interactions. Wigner distinguished between two observable manifestations of electric charge: the selection rules following from its conservation and the electromagnetic interaction of charged particles. The first phenomenon could be regarded as analogous to the conservation of "heavy particles" (i.e. nucleons, V-particles) leading to a hypothetical conserved "neutronic charge", but it remained to be seen whether also the second manifestation of electric charge found an analogue: "if this analogy [between neutronic and electric charge] has to hold, the mesonic interaction of all heavy particles must be the same, apart from the sign", so that for example "the mesonic charge of the V particles should also be equal to that of the proton" (Wigner 1952, 450). Wigner introduced a terminological distinction ("neutronic charge" vs. "mesonic charge") to provisionally keep apart the two particle properties defined by different operational procedures (conservation of heavy particles vs. constant mesonic couplings) and about whose identity only experiment would say the final word. Here Wigner made a highly refined use of various elements of the SIC-triangle to interpret experimental results and also to reflect on the possible physical significance of the formal results. 


\subsection{Oneda (1952): the characteristic number $\lambda$}

While Wigner reflected on an abstract level on the implications of the new experimental and theoretical developments, other authors continued applying the "chemical" approach. The Japanese physicist Sadao Oneda, building upon previous work by himself, Pais, Wigner and others, published a "Note on the law of conservation of heavy particles" (1952). Oneda began by noting experimental evidence in favour of proton stability and heavy particle conservation, and proposed to explain these phenomena by means of an "ad hoc assumption": "In every possible interaction scheme between stable elementary particles, the difference of the number of protons and antiprotons should be conserved" (Oneda 1952, 568). On this basis, unstable particles could be classified by assigning to each particle a "characteristic number $\lambda$ " with $\lambda=\mathrm{m}$ (number of protons) - $\mathrm{n}$ (number of antiprotons) among the particle's decay products. Oneda did not regard $\lambda$ as an intrinsic, physically conserved particle property, though:

"To our regret, the deeper cause for the conservation of heavy particles is unknown. The characteristic number $\lambda$ may be hoped to be attributed to some intrinsic properties of elementary particles. Indeed, Wigner's proposition seems to interpret the conservation of $\lambda$ as the conservation of, as it were, neutronic or mesic charge, and Tiomno and Yang referred to this principle in their treatment of reflection properties of spin $1 / 2$ particle" (Oneda 1952, 568, italics in the original).

The bottom-up approach of classification of particle phenomena in terms of "selection rules" had led to a satisfactory mathematical formalism and shown possible connections to conserved quantities. However, Oneda, like Pais and Wigner, perceived a gap between a purely mathematical conservation like that of the parameter $\lambda$ and a physically conserved quantity conceived in analogy to electric charge, showing how the heuristic power of the SIC-triangle cannot be reduced to an "application" of mathematical invariances.

\section{Outlook on further developments: Hara et al.'s (1953) phase invariance, Pais' conservation of "baryons" (1953) and the rise of symmetry}

In 1953 a group of theorists from Nagoya University (Osamu Hara, Toshio Marumori, Yoshio Ohnuki and Hajime Shimodara) published a note "On the conservation of heavy particles" (1953) in which they embedded Oneda's results in their own theoretical framework of a "spinor Urmaterie" from which all particles and interactions could be derived. Within their formalism an observable $\theta$ could be constructed which was different from mass and spin, but commuted with them and therefore implied that "elementary particles possess a structure constant other than the spin and the rest mass" (Hara et al. 1953, 115). The observable $\theta$ had values +1 and -1 and the authors stated that 
it corresponded to Oneda's $\lambda$. They continued:

"[I]t would be natural to interpret it as mesic charge, and to assume the invariance of the theory under the 'gauge transformation' performed in a relation with it:

$\psi \rightarrow \psi \exp (i \theta \alpha), \psi^{*} \rightarrow \psi^{*} \exp (-\mathrm{i} \theta \alpha)$

where $a$ is an arbitrary constant. From this requirement follows the conservation law of mesic charge which, in our opinion, is nothing but the conservation of heavy particles. Thus, the conservation of heavy particles seems to be a strong evidence for the existence of a new intrinsic structure in spinor particles" (Hara et al 1953, 115, italics in the original).

This mathematical expression corresponds exactly to the one used today for baryon number invariance. Hara et al. linked it to the conservation of a new intrinsic property of particles, but they, too, identified this property with the "mesic charge" behind strong interactions. In Hara et al. as in Oneda's work, the full SIC-triangle was exploited to construct from observed and non-observed phenomena a constant of motion and a relevant invariance which could be tentatively linked to a new physically conserved quantity, whose existence was assumed independently in analogy to electric charge.

By the early 1950s theorists were responding at increasing pace to experimental developments and to other theorists' suggestions, and the "conservation of heavy particles" was often discussed as part of broader theoretical schemes such as Hara et al.'s "spinor Urmaterie". It would be impossible to summarize here all further developments regarding that issue and I shall conclude my overview by discussing a paper by Abraham Pais which serves as an example of how different heuristic approaches could be combined to embed the conservation of heavy particles in elaborate mathematical models with a multi-layered physical interpretation. Pais began his paper by proposing a new term for what had until then been called "heavy particles":

"Experiments tell us that we can no longer talk about conservation of nucleons only but that by heavy particles one has to understand the totality of at least nucleons and $\mathrm{V}_{1^{-}}$ particles. Without prejudging on the relationship between the $\mathrm{V}_{1}$ and the nucleon it seems practical to have a collective name for these particles and other which possibly may still be discovered and which may also have to be taken along in the conservation principle just mentioned. It is proposed to use the fitting name 'baryon' for this purpose" (Pais 1953, 457).

A "baryon" was therefore defined "chemically" as a (known or unknown) particle participating of a selection rule leading to overall number conservation. It is not possible to describe here all features of Pais' model, but its core idea was to represent all "baryons" by means of one field which depended both on space-time coordinates and on additional degrees of freedom in a threedimensional " $\omega$-space”. States of this field corresponding to certain values of angular momentum and parity in $\omega$-space were interpreted as different particles (e.g. nucleons, V-particles). Pais 
indicated these states with the symbols of atomic spectroscopy: ${ }^{2} S_{1 / 2}$ for nucleons, $P$ for the $\mathrm{V}_{0}$ and so on (Pais 1953, 459). Having written the equation for the field dynamics, Pais listed its invariances and linked them to known conservations and selection rules for baryon decays and interactions. What about the conservation of baryons? Pais associated it to an invariance of the interaction with respect to a phase transformation acting on baryon fields $(\psi)$ which left meson $(\varphi)$ and photon (A) fields unchanged:

"Phase invariance:

$\psi=\exp (\mathrm{ia}) \psi^{\prime} ; \psi$ bar $\rightarrow \exp (-\mathrm{ia}) \psi \mathrm{bar} ; \varphi=\varphi^{\prime} ; \mathrm{A}_{\mathrm{i}}=\mathrm{A}_{\mathrm{i}}{ }^{\prime}$

[...] Here $a$ is a constant. The corresponding conservation law [...] corresponds to the conservation of baryons." (Pais 1953, 466).

Pais here did not associate the invariance to a parameter " $\lambda$ " or " $\theta$ ", as his Japanese colleagues had done, but simply assumed that the arbitrary phase $a$ would be multiplied by \pm 1 for baryons/antibaryons and by 0 for all other particles. No other numerical values for the factor multiplying the parameter $a$ were taken into consideration, although there was no formal reason not to do so. Since these values correspond to the assignments of "baryon number" used today, one might at first regard Pais' choice as unproblematic, but at the time the decision of not introducing one symbol to generally represent the different values is indicative of the fact that Pais did not conceive the number multiplying $a$ as representing new physical degree of freedom. The different treatment of the "anonymous" parameter and of coordinates in $\omega$-space gives an example of how Pais had a multi-layered picture of the epistemic significance of mathematical invariances: When he applied group theory to develop a model of baryon-meson interactions he was not guided by abstract mathematical considerations, but was rather using formal tools to develop a physical analogy between particles and atomic/nuclear structure. About the conservation of baryons he wrote that it was "so far insufficiently dealt with from a physical point of view" (Pais 1953, 467). In all this, the SIC-triangle was a fundamental heuristic tool allowing him to connect experimental results both to mathematical invariances and to physical analogies.

Pais' $\omega$-space formalism soon became obsolete, but the name "baryon" stuck, although it would take some time before the physics community reached an agreement on the existence and nature of the physical quantity which might be associated to baryon conservation. The earliest occurrence I could trace of the term "baryon number" as indicating an intrinsic property of particles - and not only the number of baryons - was in Murray Gell-Mann and Arthur Rosenfeld's widelyread summary of particle properties on the basis of the latest experimental data (Gell-Mann and Rosenfeld 1957, 415-416). By 1960, the conservation of baryon number and its associated phase invariance were taken up in Melvin's overview on "Elementary particles and symmetry principles" 
which was quoted in section 3 above.

\section{Summary and conclusions}

During the 1950s new heuristic strategies were developed in high-energy physics which eventually led to the emergence of theories embedding refined group theoretical structures. Physicists' reminiscences and philosophical studies have interpreted these developments in terms of historical actors "applying" mathematical invariances to theory construction. Yet this interpretation does not take into account how theories and phenomena are co-constructed and oversimplifies the process of interfacing theoretical and experimental practices. In particular, this view conflates the formation of concepts of new intrinsic degrees of freedom of elementary particles, such as baryon number, with the formulation of models containing invariances with respect to those degrees of freedom. The case study presented has clearly shown that, in the case of baryon number, the two processes were distinct and the idea of a new intrinsic degree of freedom established itself only very slowly and mainly thanks to the physical analogy to electric charge. When an invariance was associated to it, it was initially a local gauge symmetry analogous to that of electromagnetism and only later on the invariance we know today emerged, although it was at first regarded as devoid of physical significance, as it did not seem to correspond to any already established physical notions.

The analysis offered support to the thesis expounded in section 3 that mathematical invariances were only one element of a broader heuristic complex in which the notions of selection rules and physically conserved quantities were central. I labelled the relevant constellation of heuristic strategies the "SIC-triangle" (fig. 1) and in the case study we have met examples of different heuristic strategies from it. Allowing for some generalization we may sum them up as follows:

- through the formalization of positive (observation) and negative (non-observation) experimental results in terms of selection rules, patterns among experimental data were constructed (Michel 1950b, Schiff 1952, Pais 1952, Oneda 1952). The development of a phenomenologically oriented, "chemical" approach to conceptualizing experimental evidence in such terms was a key premise for exploiting the paths of the SIC-triangle by associating a selection rule to an invariance and/or to a (new) physically conserved quantity. However, most authors were not ready to regard a chemically derived parameter as representing a fully new intrinsic property. The first ones to explicitly contemplate such a possibility in the case of baryon number were Wigner (1952) and Zeldovich (1952). However, Wigner also always underscored the gap between formal mathematical invariances and conservation laws and physical invariance principles and conserved 
quantities (Wigner 1949, Wick, Wightman\&Wigner 1952);

- $\quad$ starting from an analogy to an already existing physically conserved quantity (in the case study usually electric charge) particle phenomena could be conceptualized in terms of new "substance-like" (partially) conserved properties. In the case of baryon number, this was the charge of "heavy-particle" (strong) interactions. From the existence of such a quantity, one could in turn construct models with new invariances and/or derive testable selection rules (Weyl 1929, Stückelberg 1938a,b, Okayama 1948, Wigner 1949, Jordan 1952);

- mathematical invariances and, more in general, group-theoretical methods were employed in model-building already before 1950. Once a model with an invariance was postulated, selection rules could be derived and the existence of a relevant physically conserved quantity could be tentatively assumed (Michel 1950a, Yang\&Tiomno 1950, Hara et al. 1953, Pais 1952, 1953). However, we noted how group-theoretical methods were usually applied only to well-established physical degrees of freedom such as space-time, electric and "strong" charge or (from ca. 1952 onward) isospin. Instead, there seem to have been in the early phase a certain restraint in applying complex mathematical method to the manipulations of "chemically" derived parameters.

In conclusion, Weinberg's suggestion that one may "observe equalities of rates and masses" and from there directly infer the existence of a mathematical invariance is an a posteriori simplification and idealization which has often been taken up by philosophers of science eager to find evidence for a special heuristic efficacy of mathematical structures. The findings of the present study instead suggest that the role mathematical invariances and group theory in the construction of scientific knowledge in early particle physics should be historically and philosophically understood as part of an epistemic complex of "symmetry heuristics" which still largely awaits investigation.

\section{Acknowledgements}

The research presented here is part of project "Exploring the "dark ages" of particle physics: isospin, strangeness and the construction of physical-mathematical concepts in the pre-Standard-Model era (ca. 1950e1965)" funded by the German Research Council (DFG BO 4062/21) at the TU Berlin (Germany). It was partially supported by the ANR Research Network "Circulation of algebraic and arithmetic practices and knowledge (1870-1945)" (Ref. ANR-10-JCJC0101) and has greatly benefited from the discussions in Network workshops. I wish to thank Erhard Scholz for allowing me to profit from his knowledge on Hermann Weyl's ideas on symmetry and physics and the anonymous referee for remarks and suggestions which helped greatly improve the paper. 


\section{Bibliography}

Borrelli, A. (2009). The emergence of selection rules and their encounter with group theory: 19131927. Studies in the History and Philosophy of Modern Physics, 40: 327-337

Borrelli, A. (2011). Angular momentum between physics and mathematics. In K.-H. Schlote \& M. Schneider (Ed.), Mathematics Meets Physics (pp. 395-440). Frankfurt a. M.: Verlag Harri Deutsch

Brading, K. \& H. Brown (2003). Symmetries and Noether's theorem. In K. Brading \& E. Castellani (Ed.), Symmetries in Physics. Philosophical Reflections (pp. 89-109). Cambridge: Cambridge University Press

Brading, K. \& E. Castellani (2003). Introduction. In K. Brading \& E. Castellani (Ed.), Symmetries in Physics. Philosophical Reflections (pp. 1-18). Cambridge: Cambridge University Press

Brown, L. M. (1988). Remarks on the history of isospin. In K. Winter (Ed.), Festi-Val: Festschrift for Val Telegdi (pp 39-47). Amsterdam: Elsevier

Debs, T. A. \& M. L. G. Redhead. (2007). Objectivity, Invariance, and Convention. Symmetry in Physical Science. Cambridge MA: Harvard University Press

French, S. (1999). Models and mathematics in physics: the role of group theory. In J. Butterfeled \& C. Pagonis (Ed.), From Physics to Philosophy (pp. 187-207). Cambridge: Cambridge University Press

French, S. (2000). The reasonable effectiveness of mathematics: partial structures and the application of group theory to physics. Synthese, 125: 103-120

Gell-Mann, M. \& A. Rosenfeld (1957). Hyperons and heavy mesons (systematics and decay). Annual Review of Nuclear Science, 7: 407-478

Hara, O., T. Marumoti, Y. Ohnuki \& H. Shimodaira (1953). On the conservation of heavy particles. Progress of Theoretical Physics, 10: 114-115

Haywood, S. (2011). Symmetries and Conservation Laws in Particle Physics. London: Imperial College Press

Hon, G. \& B. R. Goldstein. (2008). From 'summetria' to Symmetry: the Making of a Revolutionary Concept. Berlin: Springer

Itzykson, C. \& J.-B. Zuber (1980). Quantum Field Theory. New York: McGraw-Hill

Jordan, P. (1952a). Uber Die Erhaltungssatze Der Physik. Zeitschrift Fur Naturforschung, A 7: 7881

Jordan, P. (1952b). Uber Die Erhaltungssatze Der Physik II. Zeitschrift Fur Naturforschung, A7: 
$701-702$

Konuma, M. (1989). Social aspects of Japanese particle physics in the 1950s, in L. Brown et al. (Ed.), Pions to Quarks: Particle Physics in the 1950s (pp. 536-548). Cambridge: Cambridge University Press

Lyre, H. (2012). Symmetrien, Strukturen, Realismus. In M. Esfeld (Ed.), Philosophie der Physik (pp 368-389). Berlin: Suhrkamp

Marx, G. (1954a). Die Wechselwirkung Der Elementarteilchen und Die Erhaltungssatze', Acta Physica Academiae Scientiarum Hungaricae, 3: 55-58

Marx, G. (1954b). Uber die Erhaltung Der Fermionenladung', Zeitschrift Fur Naturforschung, A9: $1051-1052$

McConnell, J. (1946). Production and annihilation of negative protons. Nature, 158: 280-281

Melvin, M. (1960). Elementary particles and symmetry principles. Reviews of Modern Physics, 32: $477-518$

Messiah, A. M. L. \& H. P. Noyes (Ed.) (1952). Proceedings of the Rochester Conference on meson physics, January 11-12. 1952. Rochester, New York: Dept. Physics, University of Rochester (typescript) http://www.osti.gov/bridge/purl.cover.jsp?purl=/4407802-ATR9hR/

Michel, L. (1950a). Interaction between four half-spin particles and the decay of the $\mu$-meson. Proceedings of the Physical Society A, 63: 514-531

Michel, L. (1950b). Reactions between nucleons and mesons, and the search for negative protons. Nature, 166: 654-655

Michel, L. (1989). Symmetry and conservation laws in particle physics in the fifties. In L. Brown et al. (Ed.), Pions to Quarks: Particle Physics in the 1950s (pp. 373-383). Cambridge: Cambridge University Press

Ne'eman, Y. (1987). Hadron symmetry, classification and compositeness. In M. Doncel et al. (Ed.), Symmetries in Physics 1600-1980 (pp. 499-555). Barcelona: Universitat Autònoma de Barcelona

Okayama, T. (1949). On the mesic charge. Physical Review, 75: 308

Oneda, S. (1952). Note on the law of conservation of heavy particles. Progress of Theoretical Physics, 8: 568-569

Pais, A. (1952). Some remarks on the V-particles. Physical Review, 86: 663-672

Pais, A. (1953). On the baryon-meson-photon system. Progress in Theoretical Physics, 10: 457-469

Pais, A. (1986). Inward Bound. Of Matter and Forces in the Physical World. Oxford: Oxford University Press

Schiff, L. I. (1952). Neutral V-Particle decay and the negative proton. Physical Review, 85: 374 
Schweber, S. S. (1986). The empiricist temper regnant: theoretical physics in the United States 1920-1950. Historical Studies in the Physical and Biological Sciences, 17: 55-98

Schweber, S. S. (2003). Quantum field theory. From QED to the Standard Model, in M. J. Nye (Ed.), The Cambridge History of Science. Vol. 3: The Modern Physical and Mathematical Sciences (pp 375-393). Cambridge: Cambridge University Press

Stückelberg, E.C.G. (1938a). Die Wechselwirkungskräfte in der Elektrodynamik und in der Feldtheorie der Kernkräfte. Teil I. Helvetica Physica Acta, 11: 225-244

Stückelberg, E.C.G. (1938b). Die Wechselwirkungskräfte in der Elektrodynamik und in der Feldtheorie der Kernkräfte. Teil II und III, Helvetica Physica Acta, 11: 299- 328

van Fraassen, B. (1989). Laws and Symmetry. Oxford: Oxford University Press

Weinberg, S. (2011). Symmetry: A 'key to nature's secrets', New York Review of Books October 27th, http://www.nybooks.com/articles/archives/2011/oct/27/symmetry-key-natures-secrets/ last accessed 6/25/2013

Weyl, H. (1929). Elektron und Gravitation. I. Zeitschrift für Physik A, 56: 330-352

Wick, G. C., A. Wightman \& E. Wigner. (1952). The intrinsic parity of elementary particles. Physical Review, 88: 101-105

Wigner, E. (1928). Über die Erhaltungssätze in der Quantenmechanik. Nachrichten der Gesellschaft der Wissenschaften zu Göttigen, 1927: 375-381

Wigner, E. (1949). Invariance in physical theory. Proceedings of the American Philosophical Society, 93: 521-526

Wigner, E. (1952). On the law of conservation of heavy particles. Proceedings of the National Academy of Sciences of the United States of America, 38: 449-451

Yang, C. N. \& J. Tiomno (1950). Reflection properties of spin $1 / 2$ fields and a universal Fermi-type interaction. Physical Review, 79: 495-498

Zeldovich, Y. B. (1952). On the theory of elementary particles. Conservation of the nuclear charge an a possible new type of V-particles [in Russian]. Doklady Akademii Nauk SSSR, 86: 505508. English translation in Y.B. Zeldovich, Selected Works 2 (pp. 56-60). Princeton: Princeton University Press (1993)

Zeldovich, Y. B. (1953). On the neutrino charge of elementary particles [in Russian]. Doklady Akademii Nauk SSSR, , 91: 1317-1320. English translation in Y.B. Zeldovich, Selected Works 2 (pp. 62-66). Princeton: Princeton University Press (1993) 
Figure 1.: The "SIC-triangle". Each of the corners represents an independent heuristic strategy: conceptualizing experimental results in terms of selection rules, postulating the existence of a physically conserved quantity and employing mathematical invariances in the construction of new models. Additionally, to each arrow corresponds a further heuristic strategy linking two of the previous ones: (1) finding a model with a mathematical invariance accounting for an empirically established selection rules; (2) deriving from a mathematical invariance of a model one or more selection rules to be matched to experiment; (3) associating to the mathematical invariance of a model a physically conserved quantity; (4) finding a model with a mathematical invariance accounting for a postulated or an empirically established physically conserved quantity (e.g. electric charge); (5) assuming the existence of a physically conserved quantity and deriving from it selection rules to be tested; (6) postulating the existence of a physically conserved quantity which accounts for an empirically established selection rule.

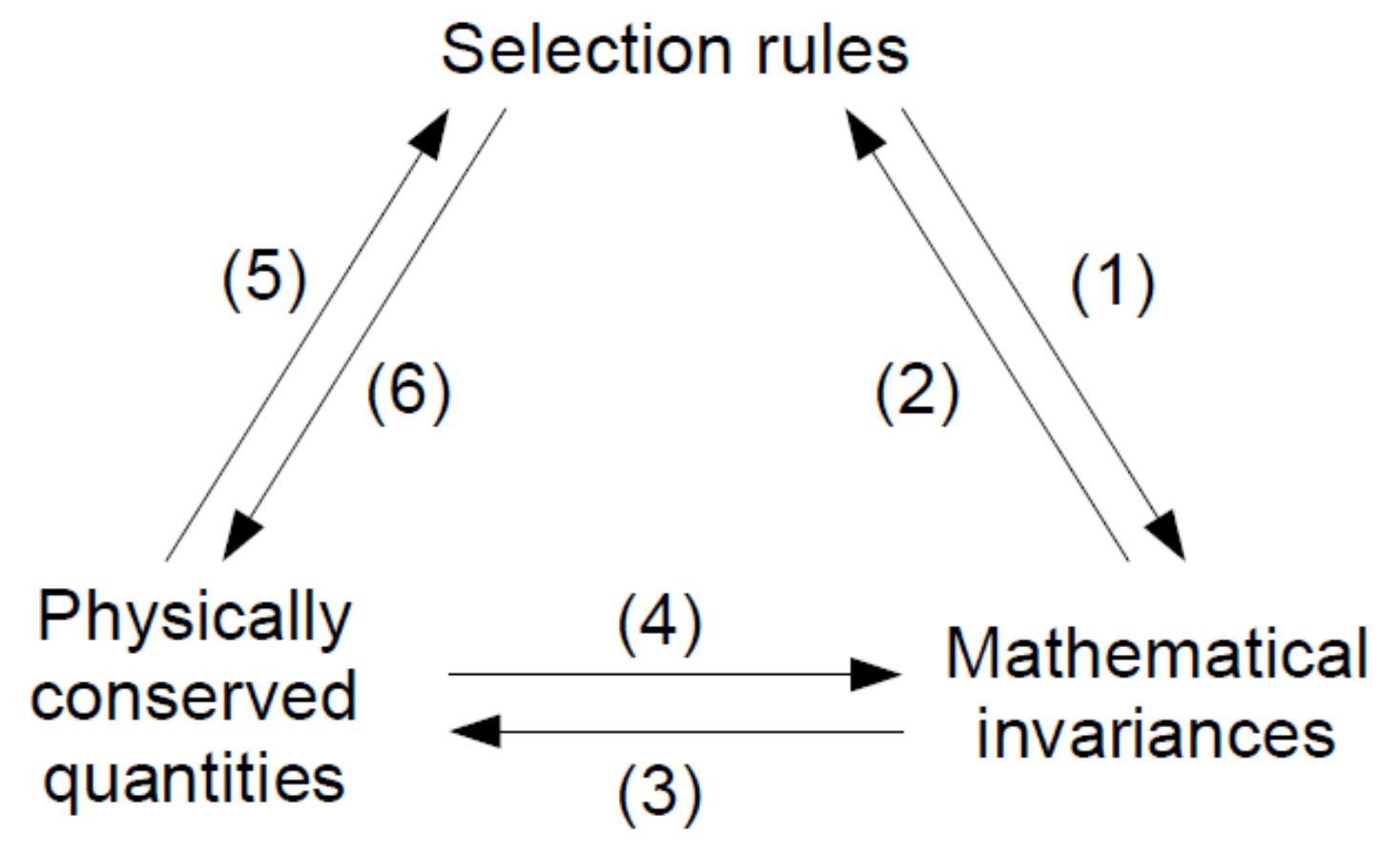

